\title{
Collapse of Incoherent Light Beams in Inertial Bulk Kerr Media
}

\author{
Ole Bang, ${ }^{1,2}$ Darran Edmundson, ${ }^{2}$ and Wieslaw Królikowski ${ }^{3}$ \\ ${ }^{1}$ Department of Mathematical Modelling, Technical University of Denmark, DK-2800 Lyngby, Denmark \\ ${ }^{2}$ Optical Sciences Centre, Research School of Physical Sciences and Engineering, Australian National University, \\ Canberra ACT 0200, Australia \\ ${ }^{3}$ Australian Photonics Cooperative Research Centre, Laser Physics Centre, Research School of Physical Sciences and Engineering, \\ Australian National University, Canberra ACT 0200, Australia
}

(Received 12 July 1999)

\begin{abstract}
We use the coherent density function theory to show that partially coherent beams are unstable and may collapse in inertial bulk Kerr media. The threshold power for collapse, and its dependence on the degree of coherence, is found analytically and checked numerically. The internal dynamics of the walk-off modes is illustrated for collapsing and diffracting partially coherent beams.
\end{abstract}

PACS numbers: 42.65.Jx, 42.65.Sf, 42.65.Tg

It was recently demonstrated by Mitchell et al. that a slow self-focusing nonlinear medium can support selftrapped partially coherent beams, or incoherent solitons $[1,2]$. Such beams are generated by an incoherent source with no correlation between light emitted from different points. This results in some level of randomness (or partial correlation) in the phase across the beam. The weaker the correlation, the stronger the incoherence [3]. As a result, a partially coherent beam spreads faster than its coherent counterpart of the same width. Furthermore, the intensity distribution across the beam exhibits speckle structure, which prevents the "standard" uniform self-focusing observed in instantaneous nonlinear media as the beam tends to form filaments. The necessary condition for soliton formation is that the nonlinear response of the medium is slow compared to the fast fluctuations of the phase front of the incident beam. In this case the medium cannot follow the random speckle structure of the beam, but responds to its time-averaged intensity.

After the early experiments in photorefractive media [1,2], Christodoulides et al. used the coherent density function theory, in which the beam is represented as a superposition of mutually incoherent components, to describe the spatially partially coherent (incoherent) beams $[4,5]$. For the special logarithmic nonlinearity the stationary incoherent soliton solution was found analytically [5]. The stationary solutions can also be found analytically using the multimode decomposition method developed by Mitchell et al. [6]. With this method, 2D (two transverse dimensions) incoherent solitons were found in logarithmic media by Christodoulides et al. [7] and 1D incoherent solitons were found in Kerr media by Carvalho et al. [8]. Recently, Snyder and Mitchell [9] and Shkunov and Anderson [10] used the geometric optics approach to find exact formulas for incoherent soliton solutions in the limit when diffraction can be neglected. This geometric optics approach is to some extent equivalent to the analysis of incoherent temporal solitons by Hasegawa [11].

Self-focusing of incoherent beams in inertial bulk Kerr media was discussed by Pasmanik [12] more than
20 years ago based on the nonlinear equation for the cross-correlation (or mutual coherence) function. This aberration-free approach indicated collapse, but led to the incorrect conclusion that the threshold power above which collapse occurs is the same as for coherent beams. Aleshkevich et al. [13] later used the mutual coherence function in a more rigorous numerical study, in which fitting to the numerical results gave an empirical formula for the effect of the partial coherence of the beam on the collapse threshold power.

Here we use the coherent density function approach to study the properties of incoherent beams in inertial bulk Kerr media. We find the first analytical formula for the collapse threshold power and show how the deterioration of spatial coherence tends to suppress self-focusing. Furthermore, we present the first fully $2 \mathrm{D}$ numerical propagation of collapsing and diffracting incoherent beams.

We consider an incoherent light beam propagating in a focusing inertial bulk Kerr medium. After a simple normalization the dynamical equation for the coherent density function $f=f(\vec{r}, z ; \vec{\theta})$ can be written in the dimensionless form (see, e.g., $[4,5]$ ),

$$
i \partial_{z} f+i \vec{\theta} \cdot \vec{\nabla}_{\perp} f+\nabla_{\perp}^{2} f+I f=0 .
$$

Here $|f|^{2}$ defines the angular intensity distribution, which is parametrized by the 2D walk-off vector $\vec{\theta}=\left(\theta_{x}, \theta_{y}\right)$. The 2D diffraction space is spanned by the transverse coordinate vector $\vec{r}=(x, y)$, with $\vec{\nabla}_{\perp}=\left(\partial_{x}, \partial_{y}\right)$, and $\nabla_{\perp}^{2}=$ $\partial_{x}^{2}+\partial_{y}^{2}$. The dimensionless total intensity $I$ is given as an integral of $|f|^{2}$ over the 2D walk-off space

$$
I(\vec{r}, z)=\int|f(\vec{r}, z ; \vec{\theta})|^{2} d \vec{\theta},
$$

where $\int d \vec{\theta} \equiv \int_{-\infty}^{\infty} \int_{-\infty}^{\infty} d \theta_{x} d \theta_{y}$. Equation (1) can be written in a simpler form without walk-off by applying the unitary transformation

$$
f(\vec{r}, z ; \vec{\theta})=F(\vec{r}, z ; \vec{\theta}) \exp \left(i \theta^{2} z / 4-i \vec{\theta} \cdot \vec{r} / 2\right),
$$


where $\theta^{2}=|\vec{\theta}|^{2}=\theta_{x}^{2}+\theta_{y}^{2}$. In terms of $F(\vec{r}, z ; \vec{\theta})$ the dynamical equations become

$$
i \partial_{z} F+\nabla^{2} F+I F=0 .
$$

The model (4) conserves the power $P_{\theta}(\vec{\theta})$ in each angular component and the Hamiltonian $H$,

$$
\begin{gathered}
P_{\theta}(\vec{\theta})=\int|f(\vec{r}, z ; \vec{\theta})|^{2} d \vec{r}, \\
H=\iint\left(|\nabla F|^{2}-\frac{1}{2} I|F|^{2}\right) d \vec{r} d \vec{\theta},
\end{gathered}
$$

where $\int d \vec{r} \equiv \int_{-\infty}^{\infty} \int_{-\infty}^{\infty} d x d y$. Also the momentum in the $x$ and $y$ direction, $M_{x, y}=\iint 2 \operatorname{Im}\left\{F^{*} \partial_{x, y} F\right\} d \vec{r} d \vec{\theta}$, the total power, $P=\int P_{\theta}(\vec{\theta}) d \vec{\theta}$, and the total momentum, $\vec{M}=\hat{x} M_{x}+\hat{y} M_{y}$, are conserved. Furthermore, the displacement of the center of mass, $\langle\vec{r}\rangle=P^{-1} \int \vec{r} I d \vec{r}$, is proportional to the total momentum, $d\langle\vec{r}\rangle / d z=\vec{M} / P$, and thus the acceleration of $\langle\vec{r}\rangle$ is zero.

To analyze the dynamics analytically we define the beam width (width of the intensity profile) or so-called virial, $V(z)=P^{-1} \int R^{2} I d \vec{r}$, where $\vec{R}=\vec{r}-\langle\vec{r}\rangle$, and $R=|\vec{R}|$ is the distance from the center of mass. Using the continuity equations for the conserved quantities we obtain for localized solutions the first derivative, $d V / d z=4 P^{-1} \iint \operatorname{Im}\left\{\vec{R} \cdot F^{*} \nabla F\right\} d \vec{r} d \vec{\theta}$, and the virial relation for the conserved acceleration,

$$
\frac{d^{2} V}{d z^{2}}=\frac{8}{P}\left(H-\frac{M^{2}}{4 P}\right)
$$

where $M^{2}=|\vec{M}|^{2}$ (see [14] for the first derivation of the virial relation for the coherent limit of the nonlinear Schrödinger (NLS) equation). If the right-hand side of (7) is negative the beam width $V(z)$ will continuously decrease and a collapse, defined as $V(z) \rightarrow 0$, will inevitably occur at a finite distance. Thus $H-M^{2} /(4 P)<0$ is a sufficient condition for collapse. Importantly, such a collapse of the total wave function implies also a collapse of each angular component, $V_{\theta}(z ; \vec{\theta})=P^{-1} \int R^{2}|F(\vec{r}, z ; \vec{\theta})|^{2} d \vec{r} \rightarrow 0$. If the right-hand side of (7) is positive, $H-M^{2} /(4 P)>0$, we have to do further analysis to determine whether a collapse can occur or not. For example, if the beam is given a sufficiently strong focusing at the input $[d V(0) / d z<0]$ a collapse could, in principle, develop despite the second derivative of the virial being constant and positive.

We now assume that the profiles of the intensity and the angular power spectrum are both radially symmetric and Gaussian at the input $(z=0)$, i.e.,

$$
f(\vec{r}, z=0 ; \vec{\theta})=\sqrt{\frac{I_{0}}{\pi \sigma^{2}}} \exp \left(-\frac{\theta^{2}}{2 \sigma^{2}}-\frac{r^{2}}{2 \Delta^{2}}\right),
$$

where $I_{0}$ is the peak intensity $[I(\vec{r}, z=0)=$ $\left.I_{0} \exp \left(-r^{2} / \Delta^{2}\right)\right]$. This is the so-called Gaussian-Schell model of partially coherent light [3]. In logarithmic media, such a beam will maintain its Gaussian statistics during propagation [5]. In inertial Kerr media this is not so. Transforming this initial condition according to Eq. (3), the total power and the Hamiltonian become $P=\pi \Delta^{2} I_{0}$ and $H=\pi I_{0}+\pi \Delta^{2} \sigma^{2} I_{0} / 4-\pi \Delta^{2} I_{0}^{2} / 4$, respectively, with the momentum being zero, $M=0$, due to symmetry. From Eq. (7) we then obtain that the beam will collapse if the total power is above the threshold $(H<0)$,

$$
P_{\mathrm{th}}=P_{\mathrm{th}}^{\mathrm{NLS}}(1+\beta),
$$

where $\beta=\Delta^{2} \sigma^{2} / 4$ is the number of speckles inside the beam cross section, and $P_{\mathrm{th}}^{\mathrm{NLS}}=4 \pi$ is the threshold power for collapse of coherent Gaussian beams described by the NLS equation [15]. Importantly $d V(0) / d z=0$ for this initial condition, and thus $P>P_{\text {th }}$, is both a necessary and sufficient condition for a total collapse in the sense that the total beamwidth goes to zero, $V(z) \rightarrow 0$. As could be expected, the power threshold for collapse increases with the degree of incoherence $\beta$. In other words, the more incoherent the beam is the stronger is the tendency to diffract, and the more power is required for the self-focusing effect to dominate diffraction and thereby cause a collapse to occur. The expression (9) represents the first analytical prediction of the influence of the degree of incoherence on the self-focusing of coherent beams in inertial Kerr media and agrees well with the empirical formulas $P_{\mathrm{th}}=P_{\mathrm{th}}^{\mathrm{NLS}}(1+0.6 \beta)$ obtained numerically by Aleshkevich et al. [13]. Note that in the coherent limit when $\sigma \rightarrow 0$ and the angular spectrum becomes a delta function, the power threshold reduces to the NLS threshold, $P_{\mathrm{th}}^{\mathrm{NLS}}$, as it should.

To study the incoherent beam propagation numerically we discretize the walk-off space, $F(\vec{r}, z ; \vec{\theta}) \rightarrow F_{n}(\vec{r}, z)$, into a central component $(n=1)$ at $\theta_{x}=\theta_{y}=0$, and $N_{r}$ rings each with $N_{s}$ equispaced spokes or components on them, as illustrated in Fig. 1. Thus the forward traveling central component has zero walk-off and all components on a given ring walk off at the same angle to the $z$ axis, but a different angle in the $(x, y)$ plane. This gives the discrete set of equations,

$$
i \partial_{z} F_{n}+\nabla^{2} F_{n}+I F_{n}=0
$$

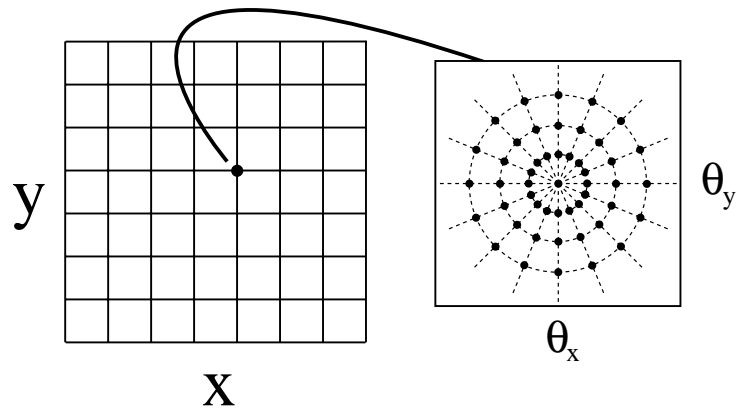

FIG. 1. Discretization of the $\vec{\theta}$ walk-off space into a central component $(n=1)$ at $\theta_{x}=\theta_{y}=0$ and $N_{r}$ rings, each with $N_{s}$ equispaced spokes or components on them. 


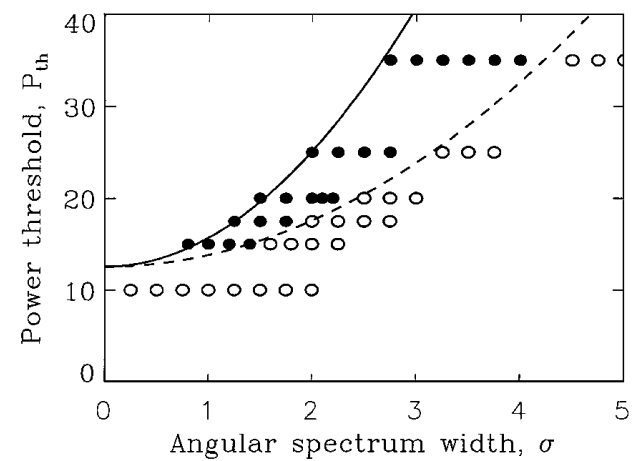

FIG. 2. Analytical collapse threshold $P_{\mathrm{th}}^{\mathrm{NLS}}(1+\beta)$ (solid line) and best numerical fit $P_{\text {th }}^{\mathrm{NLS}}(1+0.4 \beta)$ (dashed line) versus the angular spectrum width $\sigma$. Filled (open) circles indicate numerically observed collapse (diffraction).

where the intensity is now a sum over $N$ components

$$
I=\sum_{n=1}^{N} \Omega_{n}\left|F_{n}\right|^{2}, \quad N=N_{r} N_{s}+1,
$$

weighted with the solid angle $\Omega_{n}$, which denotes the resolution in $\vec{\theta}$ space (dependent on the ring number). The discrete model (10) with $I \rightarrow \ln I$ was used by Christodoulides et al. to study incoherent 1D solitons in logarithmic media [5]. The mathematical problem of collapse in Eq. (10) was studied by Bergé [16].

We have fixed $\Delta=1$ and used the split-step Fourier technique to solve Eq. (10) with $N=513$ walk-off components $\left(N_{s}=2 N_{r}=32\right)$ and $128 \times 128$ equidistant points in a square $(x, y)$ lattice over $[-5,5]$. Furthermore, we have fixed $\theta_{m} / \sigma=2.45$, where $\theta_{m}$ is the maximum walk-off ( $\theta$ value on the outer ring 16), which defines

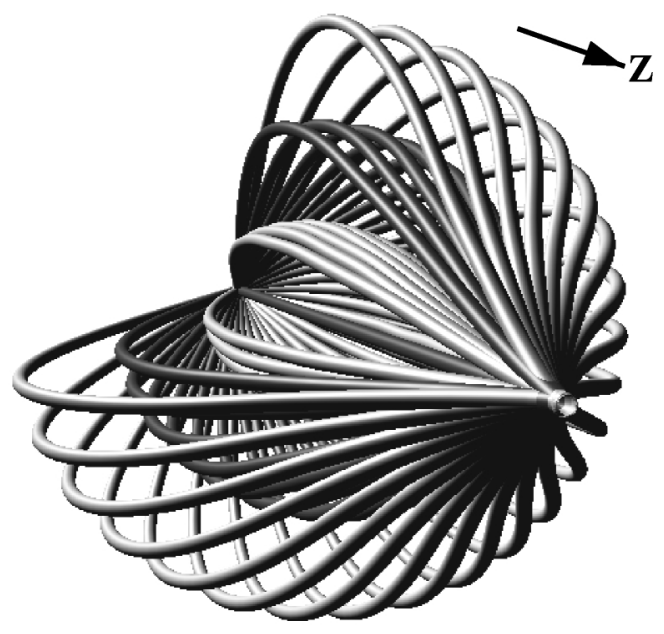

FIG. 3. Evolution of the position of the peak amplitude of the individual components, shown as tubes, during a collapse for $\sigma=1.75$ and $P=20$. Shown is the central component along with components from rings 2,4 , and 6 , having respective walk-offs $\theta=0,0.5,1.1$, and 1.6. A quadrant of components has been removed in order to see the internal structure.
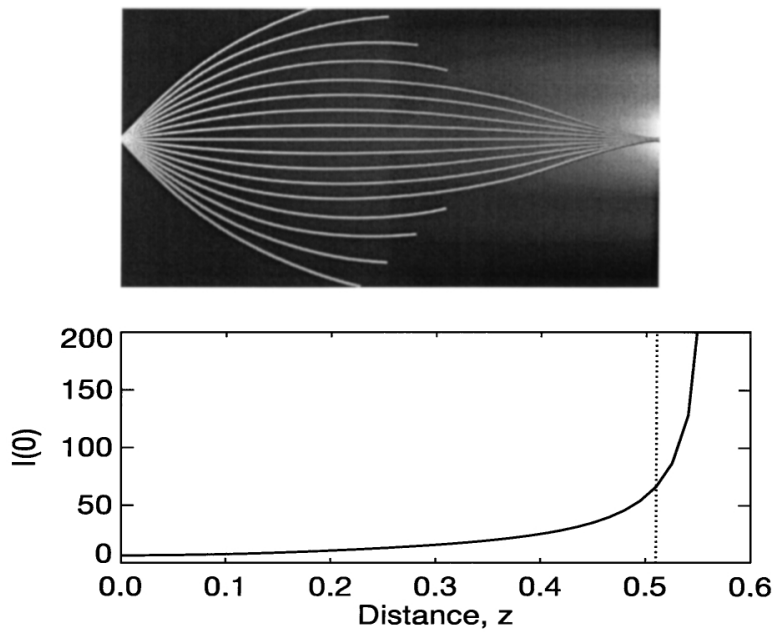

FIG. 4. Top: components from a longitudinal cut plane of Fig. 3 superimposed on the total intensity in grey scale (white being the most intense). Shown is the central component and components from rings $2,4, \ldots$, and 16 . Bottom: evolution of the total peak intensity at $\vec{r}=(0,0)$.

the cone of angles, in which the walk-off components are distributed. The numerical results, which are summarized in Fig. 2, confirm the analytically predicted dependence of the collapse threshold on the degree of coherence.

The discrepancy between the best fit $P_{\mathrm{th}}=P_{\mathrm{th}}^{\mathrm{NLS}}(1+$ $0.4 \beta$ ) and the analytical prediction (9) seems to be an artifact of the finite number of walk-off components, which should cause the collapse threshold to saturate for large values of $\sigma$ (i.e., for strongly incoherent beams), as observed for two coupled NLS equations [17]. However, it is not computationally feasible to prove this.

In general each component always starts to walk off at an angle $A \tan (\theta)$, corresponding to simple linear walkoff. Depending on the power and the value of $\theta$ for the

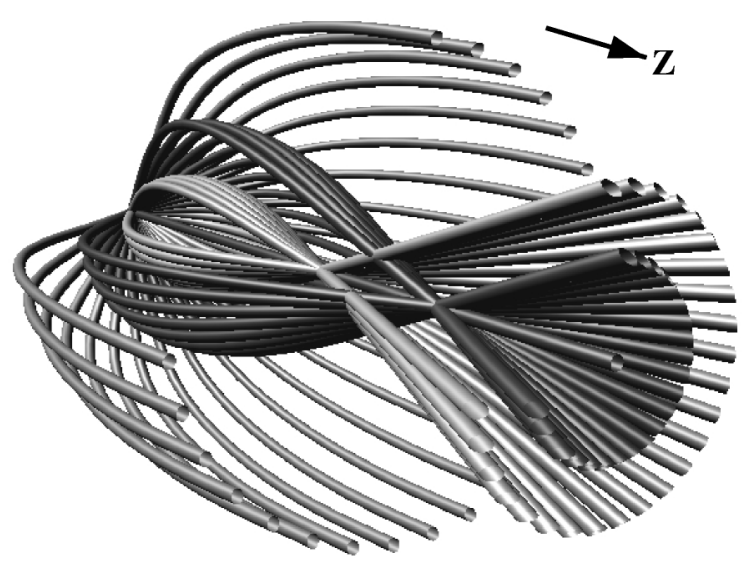

FIG. 5. Evolution of the center of mass of the individual components, shown as tubes, during diffraction for $\sigma=2.75$ and $P=20$. Shown is the central component along with components from rings 2,4 , and 6 , having respective walk-offs $\theta=0$, $0.8,1.7$, and 2.5. A quadrant of components has been removed in order to see the internal structure. 

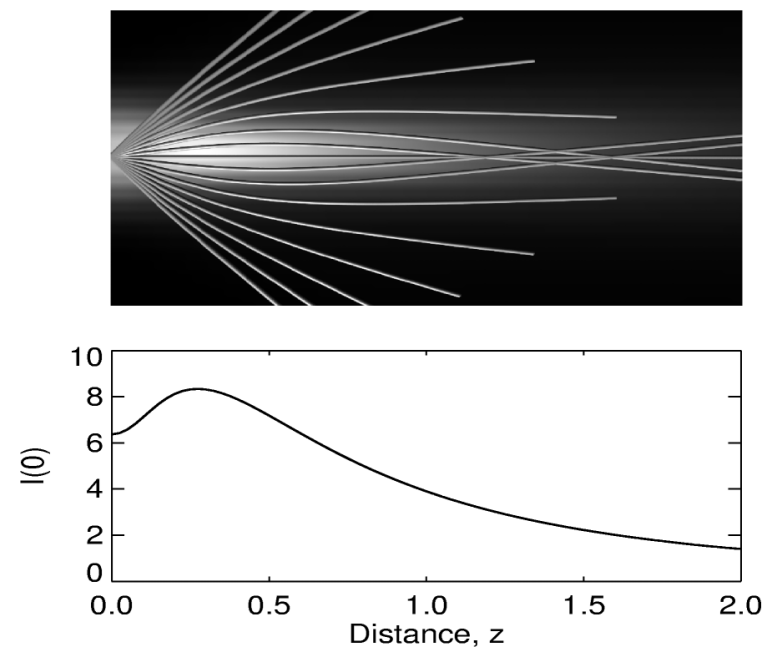

FIG. 6. Top: components from a longitudinal cut plane of Fig. 5 superimposed on the total intensity in grey scale (white being the most intense). Shown is the central component and components from rings $2,4, \ldots$, and 16 . Bottom: evolution of the total peak intensity at $\vec{r}=(0,0)$.

particular component, a certain part of the field escapes the attractive potential (or waveguide) of the total intensity and continues to walk off, while another part is captured and reflected back towards the center. If enough power is reflected a collapse occurs with the intensity going to infinity at the center $r=0$, as shown in Figs. 3 and 4 . In the waveguide analogy the waveguide created by the total intensity becomes progressively narrower during a collapse, thereby focusing all of the trapped components at the same single point. Note that the shading of the tubes has no meaning, but is used only to improve visualization.

If the reflected power is not enough to initiate a collapse, then all components will diffract and the total beam will spread out, as shown in Figs. 5 and 6. Still the components with a sufficiently small walk-off are trapped in the waveguide created by the total intensity. However, since the waveguide is now expanding, the reflected components cross the axis at different points.

While plotting the evolution of the position of the components as tubes is conceptually nice it does not show the quite complicated underlying field structure. This always consists of a trapped part and a radiative part that walks off, thereby pulling the center of mass away from the center $r=0$. Thus, plotting the position of the center of mass in Figs. 3 and 4 would not, for example, have conveyed the impression of a total collapse of all components at the same point at the center. A detailed study of the mode structure is under way.

In conclusion we have used the coherent density approach to derive an analytical expression for the power threshold for collapse of spatially partially coherent beams in inertial bulk Kerr media. We find that the necessary power for collapse increases with the degree of incoherence $\beta$ as $P_{\mathrm{th}}^{\mathrm{NLS}}(1+\beta)$, where $P_{\mathrm{th}}^{\mathrm{NLS}}$ is the threshold power for collapse of coherent beams. We have confirmed this dependence numerically and illustrated the typical beam evolution during a collapse and diffraction. Our results agree well with the threshold power $P_{\mathrm{th}}^{\mathrm{NLS}}(1+0.6 \beta)$ obtained earlier from a purely numerical analysis of the mutual coherence function.

O. Bang acknowledges support from the Danish Technical Research Council (Talent Grant No. 9800400). D. Edmundson is supported by the Natural Sciences and Engineering Research Council of Canada.

[1] M. Mitchell, Z. Chen, M. F. Shih, and M. Segev, Phys. Rev. Lett. 77, 490 (1996).

[2] M. Mitchell and M. Segev, Nature (London) 387, 880 (1997).

[3] L. Mandel and E. Wolf, Optical Coherence and Quantum Optics (Cambridge University Press, Cambridge, England, 1995).

[4] D. N. Christodoulides, T.H. Coskun, M. Mitchell, and M. Segev, Phys. Rev. Lett. 78, 646 (1997).

[5] D. N. Christodoulides, T. H. Coskun, and R. I. Joseph, Opt. Lett. 22, 1080 (1997).

[6] M. Mitchell, M. Segev, T.H. Coskun, and D.N. Christodoulides, Phys. Rev. Lett. 79, 4990 (1997).

[7] D. N. Christodoulides, T.H. Coskun, M. Mitchell, and M. Segev, Phys. Rev. Lett. 80, 2310 (1998).

[8] M. I. Carvalho, T.H. Coskun, D. N. Christodoulides, M. Mitchell, and M. Segev, Phys. Rev. E 59, 1193 (1999); see also V. A. Vysloukh et al., Sov. J. Quantum Electron. 27, 843 (1997); V. Kutuzov et al., Phys. Rev. E 57, 6056 (1998).

[9] A. W. Snyder and D. J. Mitchell, Phys. Rev. Lett. 80, 1422 (1998).

[10] V. V. Shkunov and D.Z. Anderson, Phys. Rev. Lett. 81, 2683 (1998).

[11] A. Hasegawa, Phys. Fluids 18, 77 (1975); Phys. Fluids 20, 2155 (1977); Opt. Lett. 5, 416 (1980).

[12] G. A. Pasmanik, Zh. Eksp. Teor. Fiz. 66, 490 (1974) [Sov. Phys. JETP 39, 234 (1974)].

[13] V. A. Aleshkevich, S. S. Lebedev, and A. N. Matveev, Sov. J. Quantum Electron. 11, 647 (1981).

[14] S. N. Vlasov, V. A. Petrishchev, and V. I. Talanov, Izv. Vuz. Radiofiz. 14, 1353 (1971) [Radiophys. Quantum Electron. 14, 1062 (1974)].

[15] M. I. Weinstein, Commun. Math. Phys. 87, 567 (1983).

[16] L. Bergé, Phys. Rev. E 58, 6606 (1998).

[17] O. Bang, L. Bergé, and J. J. Rasmussen, Phys. Rev. E 59, 4600 (1999); L. Bergé, O. Bang, and W. Królikowski, Report No. patt-sol/9909007. 